\title{
Prone positioning for ARDS patients-tips for preparation and use during the COVID-19 pandemic
}

\author{
Ken Kuljit S. Parhar, MD, MSc (D) Danny J. Zuege, MD, MSc - Karen Shariff, MN • Gwen Knight, MA • \\ Sean M. Bagshaw, MD, MSc
}

Received: 10 June 2020/Revised: 28 October 2020/Accepted: 29 October 2020/Published online: 24 December 2020

(C) Canadian Anesthesiologists' Society 2020

\begin{abstract}
Many patients with coronavirus disease (COVID-19) will develop acute respiratory distress syndrome (ARDS). Prone positioning is an important non-pharmacologic strategy that should be considered for all invasively ventilated patients with moderate to severe ARDS (including those with COVID-19). Prone positioning offers several physiologic and clinical benefits, including improving hypoxemia, matching ventilation with perfusion, reducing regional hyperinflation, and improving survival. To safely offer prone positioning, appropriate training, simulation, and health system planning should be undertaken. In this review, we offer ten tips, based on the Alberta provincial prone positioning strategy during COVID-19, to safely implement and improve the appropriate use of prone positioning. We provide special considerations for its use during the COVID-19 pandemic or future respiratory pandemics.
\end{abstract}

Supplementary information The online version of this article (https://doi.org/10.1007/s12630-020-01885-0) contains supplementary material, which is available to authorized users.

\section{K. K. S. Parhar, MD, MSc ( $\bowtie)$}

Department of Critical Care Medicine, University of Calgary and Alberta Health Services, Foothills Medical Center, Calgary, AB, Canada

e-mail: ken.parhar@albertahealthservices.ca

Department of Critical Care Medicine, University of Calgary, ICU Administration - Ground Floor - McCaig Tower, Foothills Medical Center, 3134 Hospital Drive NW, Calgary, AB T2N $5 \mathrm{~A} 1$, Canada

D. J. Zuege, MD, MSc · K. Shariff, MN

Department of Critical Care Medicine, University of Calgary and Alberta Health Services, Foothills Medical Center, Calgary, AB, Canada
Résumé De nombreux patients atteints de la maladie du coronavirus (COVID-19) développeront un syndrome de détresse respiratoire aiguë (SDRA, ARDS en anglais). Le positionnement ventral est une importante stratégie non pharmacologique qui devrait être envisagée pour tous les patients ventilés de manière invasive et souffrant d'un SDRA modéré à grave (y compris ceux atteints de la COVID-19). Le positionnement ventral offre plusieurs avantages physiologiques et cliniques, notamment l'amélioration de l'hypoxémie, une adéquation de la ventilation avec la perfusion, la réduction de l'hyperinflation régionale et l'amélioration de la survie. Pour offrir un positionnement ventral en toute sécurité, une formation, des simulations et une planification des ressources appropriées devraient être entreprises. Dans le cadre de ce compte rendu, nous proposons dix conseils, fondés sur la stratégie provinciale de positionnement ventral de l'Alberta au cours de la COVID-19, afin de mettre en cuvre et d'améliorer en toute sécurité l'utilisation appropriée du positionnement ventral. Nous

Critical Care Strategic Clinical Network, Alberta Health Services, Edmonton, AB, Canada

G. Knight, MA

Department of Critical Care Medicine, University of Calgary and Alberta Health Services, Foothills Medical Center, Calgary, AB, Canada

S. M. Bagshaw, MD, MSc

Critical Care Strategic Clinical Network, Alberta Health Services, Edmonton, AB, Canada

Department of Critical Care Medicine, Faculty of Medicine and Dentistry, University of Alberta and Alberta Health Services, Edmonton, AB, Canada 
décrivons des considérations particulières pour son utilisation pendant la pandémie de COVID-19 ou les futures pandémies respiratoires.

Keywords prone positioning . acute respiratory distress syndrome (ARDS) - COVID-19 . mechanical ventilation

\section{Why use prone positioning in acute respiratory distress syndrome (ARDS) particularly during pandemics with respiratory pathogens such as coronavirus disease (COVID-19)?}

Prone positioning is an important non-pharmacologic strategy with lifesaving potential that should be considered for all invasively ventilated patients with moderate to severe ARDS. Prone positioning offers several physiologic and clinical benefits, including improved oxygenation, better matching of ventilation with perfusion, reduced risk for ventilator-induced lung injury, and improved survival (reviewed previously in detail). ${ }^{1}{ }^{3}$

Several guidelines recommend prone positioning for patients with ARDS, ${ }^{4,5}$ including World Health Organization guidelines for the management of COVID19 associated ARDS. ${ }^{6}$ Practical guidance for safely upscaling the use of prone positioning during a pandemic are limited.

Given that COVID-19 could afflict large numbers of patients and overwhelm the intensive care unit (ICU) capacity of acute care hospitals, advance preparation and scale up of capacity to prone large numbers of patients is paramount. This is particularly relevant for centres with less experience with prone position ventilation, who may be compelled to undertake this therapy during a pandemic when there is no option to transfer patients to referral centres because of overwhelmed capacity.

To safely prone invasively ventilated ARDS patients and mitigate the human resource impacts, appropriate training, simulation, and health system planning must be undertaken. In this article, we offer ten tips, based on work conducted by the Alberta Critical Care Strategic Clinical Network COVID-19 Prone Positioning working group, to safely implement and increase the appropriate use of prone positioning. We highlight special considerations for proning during the COVID-19 pandemic or future respiratory pandemics.

\section{Ten tips}

1) Develop a protocol and checklist for prone positioning
Creating a guiding protocol and checklist is crucial for appropriate training and execution of prone positioning. This document should include step by step instructions on how to safely prone and unprone invasively ventilated ARDS patients (Figure), links to videos on how to prone (e.g., Guerin et al.), ${ }^{7}$ and address frequently asked questions. As an adjunct, checklists can ensure consistency for all the tasks required during proning and unproning. The checklist should be readily accessible to front line clinicians. See example checklist in the Electronic Supplementary Material [ESM], eAppendix.

\section{2) Ensure appropriate equipment is available.}

Appropriate supplies required to prone patients (flat sheets) and prevent pressure sores and ulcers while in the prone position (gel or foam face cushions) must be available. Anticipating times where substantial numbers of patients may proned, pandemic planning committees should quantify current supplies and the acquisition of additional equipment as needed. Prone positioning is not aerosol generating; however, team members should have appropriate personal protective equipment in the event ventilator tubing becomes disconnected and aerosol generation occurs while repositioning.

\section{3) Identify the ideal size and membership of the proning team based on local context.}

Identifying team members who will participate in proning patients and defining their roles and responsibilities is important. The optimal team will depend on local context, the specific disciplines participating (e.g., nursing, respiratory therapy, physicians), their experience, and staffing availability (e.g., daytime $v s$ afterhours).

Key responsibilities include airway management, venous and arterial line management, management of bladder catheters and chest tubes, and monitoring cardiorespiratory status during the procedure. Registered respiratory therapists (RRTs) or qualified physicians should be present during position changes to manage the airway. During a pandemic, limiting members of the prone positioning team to only ICU nurses and RRTs may be challenging. Alternative members of the multidisciplinary team that can participate in proning include physiotherapists, healthcare assistants, and even patient porters or security personnel if done under appropriate supervision. Adequate training must be undertaken, ideally including group simulation. All team members must have appropriate personal protective equipment. If proning is occurring frequently during a pandemic, proning teams may be established to improve efficiency. 
Table Adverse events associated with prone positioning and mitigation strategy

Potential adverse events associated with the act of prone Mitigation strategy positioning

\begin{tabular}{ll}
\hline Removal of vascular access & Checklist and dedicated team member to monitor during repositioning \\
Removal of catheters or chest tubes & Checklist and dedicated team member to monitor during repositioning \\
Accidental extubation & Physician or respiratory therapist to monitor and lead repositioning \\
Hemodynamic instability & Checklist and dedicated team member to monitor during repositioning (potentially \\
from outside of room) & Physician or respiratory therapist to monitor during repositioning \\
Transient oxygen desaturation &
\end{tabular}

Potential adverse events associated with the management of patients in the prone position

Mitigation strategy

Pressure ulcers

Frequent $(q 2 h)$ repositioning of pressure points

Use of gel or foam face cushions

Facial edema

Endotracheal tube occlusion reverse-Trendelenburg position

Frequent $(q 2 h)$ checks of airway pressures / endotracheal tube patency

\section{4) Clearly identify indications for initiating prone positioning}

Ensuring team members are familiar with indications/ criteria for prone positioning is crucial. Prone positioning should be considered in patients who are invasively mechanically ventilated and have moderate to severe
ARDS (arterial to inspired oxygen concentration $\left[\mathrm{P}_{\mathrm{a}} \mathrm{O}_{2}\right]$ $\mathrm{F}_{\mathrm{i}} \mathrm{O}_{2}$ ] ratio $\leq 150$ while on an fraction of inspired oxygen $\left[\mathrm{F}_{\mathrm{I}} \mathrm{O}_{2}\right]$ of $\left.\geq 0.60\right)^{7,8}$ These patients should have conventional supportive therapies applied prior to prone positioning including lung protective ventilation, consideration for short-term neuromuscular blockade, and

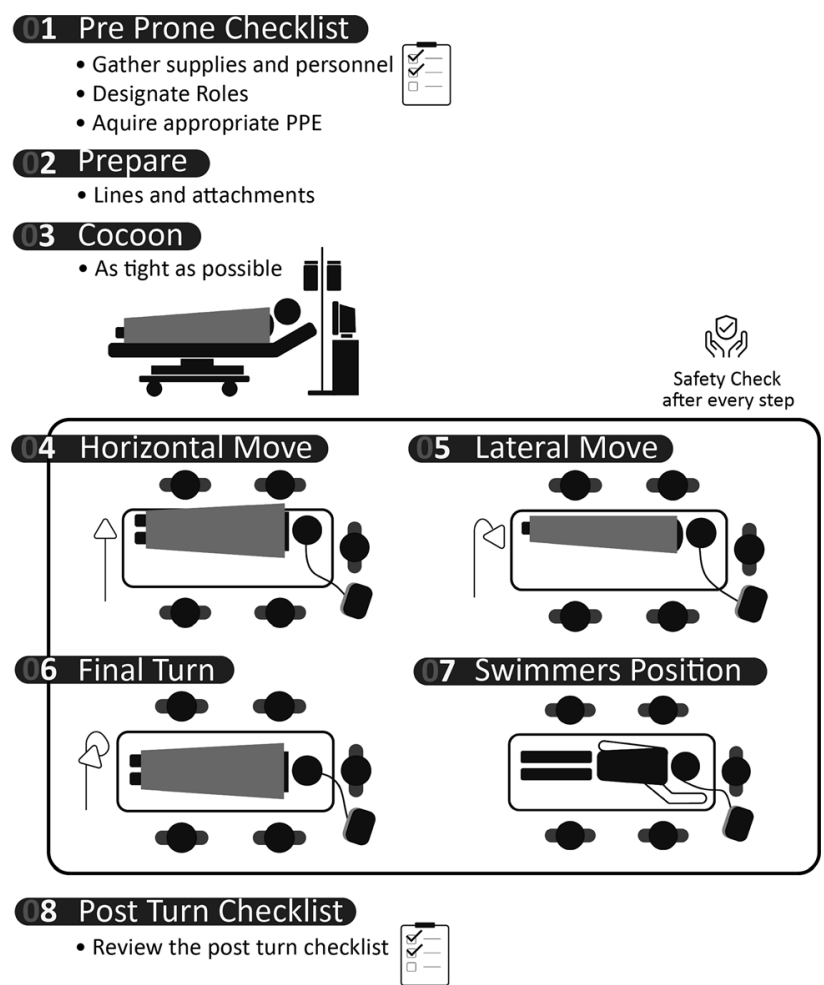

Figure Steps in prone positioning invasively ventilated patients 
exclusion of reversible causes of hypoxemia (e.g., drainage of pleural effusion or pneumothorax, diuresis of pulmonary edema).

There are few absolute contraindications to prone positioning. Pregnant women in their third trimester should not be proned routinely; however, a modified approach may be considered. ${ }^{9}$ Patients with unstable vertebral fractures, open abdomens, or open chests should not be considered for prone positioning.

Nurses, RRTs, and physicians should all be empowered to suggest and discuss the use of prone positioning during and outside of daily patient rounds. Educational interventions to improve familiarity should focus on all team members as opposed to primarily targeting physicians.

\section{5) Identify a standard time to prone and unprone patients}

Prone positioning should be incorporated into the daily ICU routine. Given that the survival benefit for prone positioning requires a minimum daily duration of $12 \mathrm{hr}$ (ideally closer to $16 \mathrm{hr}$ ), ${ }^{8}$ we suggest making a decision to prone patients by 16:00 to allow timely prone positioning while staffing is maximal during daytime hours. Patients can then be left prone positioned overnight and unproned at approximately 08:00. Using set times can facilitate high volume prone positioning during pandemic times as the same team members can prone multiple patients in succession. Patients who are acutely hypoxemic despite medical optimization can be proned in an unscheduled fashion outside of these times to avoid further deterioration. Thus, afterhours staff should be as comfortable prone positioning as daytime staff are.

\section{6) Modify care to mitigate the risk of adverse events.}

Risks can be classified as those associated with the proning maneuver and those associated with managing patients while in the prone position. Complications occurring during or immediately following proning include oxygen desaturation, loss of intravascular lines, accidental extubation, and hemodynamic instability or cardiac arrest (see Table). ${ }^{1}{ }^{3}$

Care should be modified while the patient is in the prone position. Enteral feeding should be continued while monitoring gastric residual volumes. ${ }^{10}$ To avoid pressure ulcers (especially forehead and anterior chest) frequent (e.g., every two hours) and careful rotation of the patient's limbs (e.g., shoulder) and head position are important. Facial edema can be prevented by placing the patient in reverse-Trendelenburg position while in the prone position. Pneumothoraces should be decompressed with chest tubes and should be reassessed to ensure continued decompression while prone. Interventions such as physiotherapy for passive range of motion and diagnostic tests should be scheduled for daytime hours while the patient is supine when possible.

\section{7) Utilize simulation to build competency and confidence}

Even low fidelity simulations using mannequins or team members as simulated patients can be used to help build confidence, competency, and experience in prone positioning. Simulations should ideally be conducted with the interdisciplinary team to improve communication as well as the team dynamics required for the proning maneuver. (See example low fidelity simulation in eAppendix [ESM]). Advanced simulation can incorporate transport in the prone position.

8) Assess staff barriers and facilitators to prone positioning to improve acceptability and adherence.

Formal (surveys, semi-structured interviews) or informal feedback should be sought from team members to identify barriers and facilitators to prone positioning both prior to and after the initial implementation. Feedback should be sought from all members of the multidisciplinary team participating in prone positioning.

9) Audit the use of prone positioning and provide feedback

Appropriate use of prone positioning should be routinely audited. Based on this, feedback should be provided to improve implementation and adherence. Prior to the COVID-19 pandemic, a 2018 one-day international prevalence study found the overall use of prone positioning was low despite publication of supportive clinical trials and guidance for use. ${ }^{11}$ Similarly, during the COVID-19 pandemic, the usage of prone positioning has been described as low (11-28\%). ${ }^{12}-{ }^{14}$ Targeted audit and feedback should be used to improve the rates of prone positioning in appropriate patients.

10) Consider, discuss, and plan for special circumstances.

Consider and plan for special circumstances that may arise while patients are prone positioned. The following are examples of challenging situations that can be feasibly managed:

a. Obesity is a challenge but not a contraindication to prone positioning. Appropriate pillows and supports can be placed below the patient chest and pelvis to accommodate the abdomen safely.

b. Renal replacement therapy can be provided while prone, but care must be taken to ensure vascular access is accessible and routinely assessed. This is easiest with an internal jugular dialysis catheter. 
c. If the patient has a cardiopulmonary arrest while in the prone position, cardiopulmonary resuscitation can be performed effectively in the prone position while awaiting help to unprone the patient. ${ }^{15}$

\section{Conclusions}

Prone positioning is a lifesaving non-pharmacologic strategy that should be part of the toolbox in every ICU that manages patients with ARDS. The use of prone positioning requires an understanding of indications and risks, along with appropriate health system planning through development of protocols and procedures as well as simulation and practice to gain competence and expertise.

Author contributions Ken Kuljit, S. Parhar, and Sean M. Bagshaw contributed to the conception and design of the manuscript. Ken Kuljit S. Parhar, Danny J. Zuege, Gwen Knight, Karen Shariff, and Sean M. Bagshaw wrote the manuscript.

Acknowledgements The Alberta Critical Care Strategic Clinical Network COVID-19 Prone Positioning working group was co-chaired by authors Ken Kuljit S. Parhar, Sean M. Bagshaw, and Karen Shariff. The authors would like to thank all the members of this working group along with all the critical care nurses, respiratory therapists, and physicians in Alberta for participating in interviews, completion of surveys, as well as input to develop a provincial prone positioning strategy.

Disclosures None.

Funding statement None.

Editorial responsibility This submission was handled by Dr. Sangeeta Mehta, Associate Editor, Canadian Journal of Anesthesia.

\section{References}

1. Kallet RH. A comprehensive review of prone position in ARDS. Respir Care 2015; 60: 1660-87.

2. Scholten EL, Beitler JR, Prisk GK, Malhotra A. Treatment of ARDS with prone positioning. Chest 2017; 151: 215-24.
3. Gattinoni L, Busana M, Giosa L, Macri MM, Quintel M. Prone positioning in acute respiratory distress syndrome. Semin Respir Crit Care Med 2019; 40: 94-100.

4. Chiumello D, Brochard L, Marini JJ, et al. Respiratory support in patients with acute respiratory distress syndrome: an expert opinion. Crit Care 2017; DOI: https://doi.org/10.1186/s13054017-1820-0.

5. Fan E, Del Sorbo L, Goligher EC, et al. An official American Thoracic Society/European Society of Intensive Care Medicine/ Society of Critical Care Medicine Clinical Practice Guideline: Mechanical ventilation in adult patients with acute respiratory distress syndrome. Am J Respir Crit Care Med 2017; 195: 1253-63.

6. World Health Organization. Clinical management of severe acute respiratory infection when novel coronavirus ( $\mathrm{nCoV})$ infection is suspected: interim guidance, 28 January 2020. Available from URL: https://apps.who.int/iris/handle/10665/330893 (accessed October 2020).

7. Guerin C, Reignier J, Richard JC, et al. Prone positioning in severe acute respiratory distress syndrome. N Engl J Med 2013; 368: 2159-68.

8. Munshi L, Del Sorbo L, Adhikari NK, et al. Prone position for acute respiratory distress syndrome. A systematic review and meta-analysis. Ann Am Thorac Soc 2017; 14(Supplement_4): S280-8.

9. Samanta S, Samanta $S$, Wig J, Baronia AK. How safe is the prone position in acute respiratory distress syndrome at late pregnancy? Am J Emerg Med 2014; 32(687): e1-3.

10. Mentec H, Dupont H, Bocchetti M, Cani P, Ponche F, Bleichner $G$. Upper digestive intolerance during enteral nutrition in critically ill patients: frequency, risk factors, and complications. Crit Care Med 2001; 29: 1955-61.

11. Guerin $C$, Beuret $P$, Constantin JM, et al. A prospective international observational prevalence study on prone positioning of ARDS patients: the APRONET (ARDS Prone Position Network) study. Intensive Care Med 2018; 44: 22-37.

12. Bhatraju PK, Ghassemieh BJ, Nichols M, et al. Covid-19 in critically ill patients in the Seattle region - case series. N Engl J Med 2020; 382: 2012-22.

13. Grasselli $G$, Zangrillo A, Zanella A, et al. Baseline characteristics and outcomes of 1591 patients infected with SARS-CoV-2 Admitted to ICUs of the Lombardy region. Italy. JAMA 2020; 323: $1574-81$.

14. Yang $X, Y u Y, X u J$, et al. Clinical course and outcomes of critically ill patients with SARS-CoV-2 pneumonia in Wuhan, China: a single-centered, retrospective, observational study. Lancet Respir Med 2020; 8: 475-81.

15. Mazer SP, Weisfeldt M, Bai D, et al. Reverse CPR: a pilot study of CPR in the prone position. Resuscitation 2003; 57: 279-85.

Publisher's Note Springer Nature remains neutral with regard to jurisdictional claims in published maps and institutional affiliations. 